TUM-HEP-343/99

hep-ph/9901409

January 1999

\title{
Operator Product Expansion, Renormalization Group and Weak Decays 8
}

\author{
Andrzej J. Buras \\ Technische Universität München \\ Physik Department \\ D-85748 Garching, Germany
}

\begin{abstract}
A non-technical description of the Operator Product Expansion and Renormalization Group techniques as applied to weak decays of mesons is presented. We use this opportunity to summarize briefly the present status of the next-to-leading QCD corrections to weak decays and their implications for the unitarity triangle, the ratio $\varepsilon^{\prime} / \varepsilon$, the radiative decay $B \rightarrow X_{s} \gamma$, and the rare decays $K^{+} \rightarrow \pi^{+} \nu \bar{\nu}$ and $K_{L} \rightarrow \pi^{0} \nu \bar{\nu}$.
\end{abstract}

* Dedicated to the 70th birthday of Wolfhart Zimmermann.

To appear in Recent Developments in Quantum Field Theory, Springer Verlag, eds. P. Breitenlohner, D. Maison and J. Wess. 


\section{Preface}

It is a great privilege and a great pleasure to give this talk at the symposium celebrating the 70th birthday of Wolfhart Zimmermann. The Operator Product Expansion [1] to which Wolfhart Zimmermann contributed in such an important manner [2, 3, 4] had an important impact on my research during the last 20 years. I do hope very much to give another talk on this subject in 2008 at a symposium celebrating Wolfhart Zimmermanns 80th birthday. I am convinced that OPE will play an important role in the next 10 years in the field of weak decays as it played already in almost 25 years since the pioneering applications of this very powerful method by Gaillard and Lee [5] and Altarelli and Maiani [6].

\section{Operator Product Expansion}

The basic starting point for any serious phenomenology of weak decays of hadrons is the effective weak Hamiltonian which has the following generic structure

$$
\mathcal{H}_{e f f}=\frac{G_{F}}{\sqrt{2}} \sum_{i} V_{\mathrm{CKM}}^{i} C_{i}(\mu) Q_{i}
$$

Here $G_{F}$ is the Fermi constant and $Q_{i}$ are the relevant local operators which govern the decays in question. The Cabibbo-Kobayashi-Maskawa factors $V_{C K M}^{i}$ [7, 8) and the Wilson Coefficients $C_{i}$ [1] describe the strength with which a given operator enters the Hamiltonian.

In the simplest case of the $\beta$-decay, $\mathcal{H}_{\text {eff }}$ takes the familiar form

$$
\mathcal{H}_{e f f}^{(\beta)}=\frac{G_{F}}{\sqrt{2}} \cos \theta_{c}\left[\bar{u} \gamma_{\mu}\left(1-\gamma_{5}\right) d \otimes \bar{e} \gamma^{\mu}\left(1-\gamma_{5}\right) \nu_{e}\right]
$$

where $V_{u d}$ has been expressed in terms of the Cabibbo angle. In this particular case the Wilson Coefficient is equal unity and the local operator, the object between the square brackets, is given by a product of two $V-A$ currents. This local operator is represented by the diagram (b) in fig. 11. Equation (2.2) represents the Fermi theory for $\beta$-decays as formulated by Sudarshan and Marshak [9] and Feynman and Gell-Mann [10] forty years ago, except that in (2.2) the quark language has been used and following Cabibbo a small departure of $V_{u d}$ from unity has been incorporated. In this context the basic formula (2.1) can be regarded as a generalization of the Fermi Theory to include all known quarks and leptons as well as their strong and electroweak interactions as summarized by the Standard Model. It should be stressed that the formulation of weak decays in terms of effective Hamiltonians is very suitable for the inclusion of new physics effects. We will discuss this issue briefly later on. 


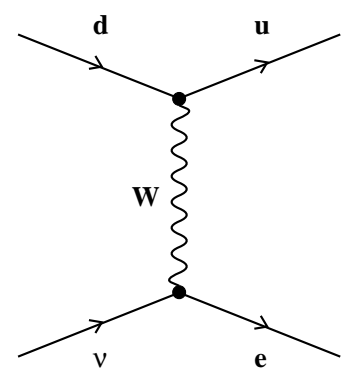

(a)

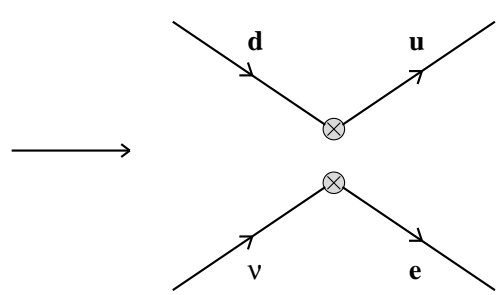

(b)

Figure 1: $\beta$-decay at the quark level in the full (a) and effective (b) theory.

Now, I am aware of the fact that the formal operator language used here is hated by experimentalists and frequently disliked by more phenomenological minded theorists. Consequently the literature on weak decays, in particular on B-meson decays [11], is governed by Feynman diagram drawings with $\mathrm{W}-$, Z- and top quark exchanges, rather than by the operators in (2.1). In the case of the $\beta$-decay we have the diagram (a) in fig. 1. Yet such Feynman diagrams with full W-propagators, Z-propagators and top-quark propagators really represent the situation at very short distance scales $\mathcal{O}\left(\mathrm{M}_{\mathrm{W}, \mathrm{Z}}, \mathrm{m}_{\mathrm{t}}\right)$, whereas the true picture of a decaying hadron with masses $\mathcal{O}\left(m_{\mathrm{b}}, m_{\mathrm{c}}, m_{K}\right)$ is more properly described by effective point-like vertices which are represented by the local operators $Q_{i}$. The Wilson coefficients $C_{i}$ can then be regarded as coupling constants associated with these effective vertices.

Thus $\mathcal{H}_{\text {eff }}$ in (2.1) is simply a series of effective vertices multiplied by effective coupling constants $C_{i}$. This series is known under the name of the operator product expansion (OPE) [1]- [4], [12]. Due to the interplay of electroweak and strong interactions the structure of the local operators (vertices) is much richer than in the case of the $\beta$-decay. They can be classified with respect to the Dirac structure, colour structure and the type of quarks and leptons relevant for a given decay. Of particular interest are the operators involving quarks only. They govern the non-leptonic decays. To be specific let us list the operators relevant for non-leptonic B-meson decays. They are:

\section{Current-Current :}

$$
Q_{1}=\left(\bar{c}_{\alpha} b_{\beta}\right)_{V-A}\left(\bar{s}_{\beta} c_{\alpha}\right)_{V-A} \quad Q_{2}=(\bar{c} b)_{V-A}(\bar{s} c)_{V-A}
$$

\section{QCD-Penguins :}

$$
Q_{3}=(\bar{s} b)_{V-A} \sum_{q=u, d, s, c, b}(\bar{q} q)_{V-A} \quad Q_{4}=\left(\bar{s}_{\alpha} b_{\beta}\right)_{V-A} \sum_{q=u, d, s, c, b}\left(\bar{q}_{\beta} q_{\alpha}\right)_{V-A}
$$




$$
Q_{5}=(\bar{s} b)_{V-A} \sum_{q=u, d, s, c, b}(\bar{q} q)_{V+A} \quad Q_{6}=\left(\bar{s}_{\alpha} b_{\beta}\right)_{V-A} \sum_{q=u, d, s, c, b}\left(\bar{q}_{\beta} q_{\alpha}\right)_{V+A}
$$

\section{Electroweak-Penguins :}

$$
\begin{array}{rlrl}
Q_{7} & =\frac{3}{2}(\bar{s} b)_{V-A} \sum_{q=u, d, s, c, b} e_{q}(\bar{q} q)_{V+A} & Q_{8} & =\frac{3}{2}\left(\bar{s}_{\alpha} b_{\beta}\right)_{V-A} \sum_{q=u, d, s, c, b} e_{q}\left(\bar{q}_{\beta} q_{\alpha}\right)_{V+A} \\
Q_{9}=\frac{3}{2}(\bar{s} b)_{V-A} \sum_{q=u, d, s, c, b} e_{q}(\bar{q} q)_{V-A} & Q_{10}=\frac{3}{2}\left(\bar{s}_{\alpha} b_{\beta}\right)_{V-A} \sum_{q=u, d, s, c, b} e_{q}\left(\bar{q}_{\beta} q_{\alpha}\right)_{V-A} .
\end{array}
$$

Here, $\alpha$ and $\beta$ are colour indices and $e_{q}$ denotes the electrical quark charges reflecting the electroweak origin of $Q_{7}, \ldots, Q_{10} . Q_{2}, Q_{3-6}$ and $Q_{7,9}$ originate in the tree level $W^{ \pm}$-exchange, gluon penguin and $\left(\gamma, Z^{0}\right)$-penguin diagrams respectively. These are the diagrams a)-c) in fig. 2. To generate $Q_{1}, Q_{8}$ and $Q_{10}$ additional gluonic exchanges are needed. The operators given above have dimension six. Of interest are also operators of dimension five which are responsible for the $B \rightarrow s \gamma$ decay. They originate in the diagram d) in fig. 2 where $\gamma$ and the gluon are on-shell. They will be given in Section 7. In what follows we will neglect the higher dimensional operators as their contributions to weak decays are marginal.
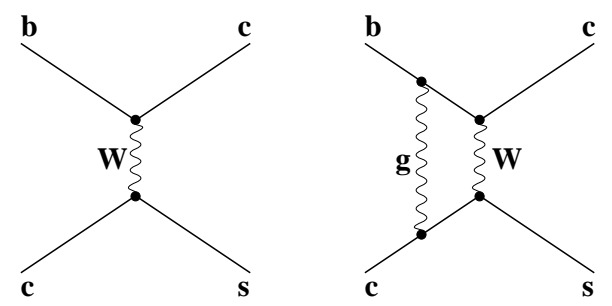

(a)

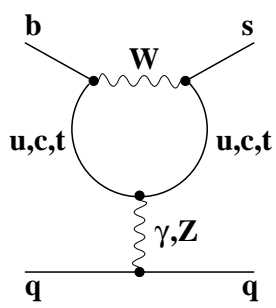

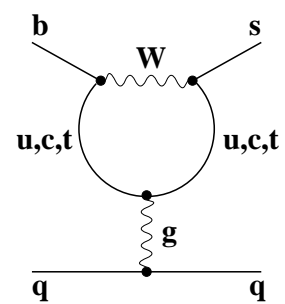

(b)

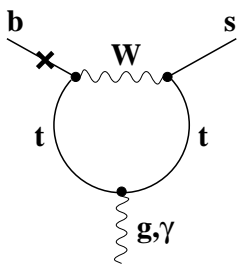

(d)

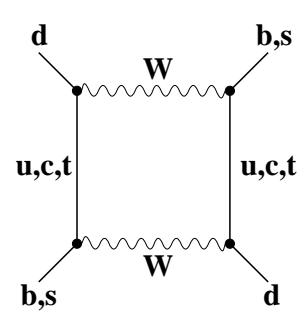

(e)

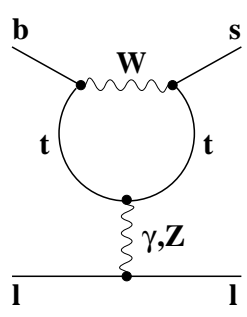

(f)

Figure 2: Typical Penguin and Box Diagrams. 
Now what about the couplings $C_{i}(\mu)$ and the scale $\mu$ ? The important point is that $C_{i}(\mu)$ summarize the physics contributions from scales higher than $\mu$ and due to asymptotic freedom of QCD they can be calculated in perturbation theory as long as $\mu$ is not too small. $C_{i}$ include the top quark contributions and contributions from other heavy particles such as W, Z-bosons and charged Higgs particles or supersymmetric particles in the supersymmetric extensions of the Standard Model. At higher orders in the electroweak coupling the neutral Higgs may also contribute. Consequently $C_{i}(\mu)$ depend generally on $m_{t}$ and also on the masses of new particles if extensions of the Standard Model are considered. This dependence can be found by evaluating the box and penguin diagrams with full $\mathrm{W}-$, Z-, top- and new particles exchanges shown in fig. 2 and properly including short distance QCD effects. The latter govern the $\mu$-dependence of the couplings $C_{i}(\mu)$.

The value of $\mu$ can be chosen arbitrarily. It serves to separate the physics contributions to a given decay amplitude into short-distance contributions at scales higher than $\mu$ and long-distance contributions corresponding to scales lower than $\mu$. It is customary to choose $\mu$ to be of the order of the mass of the decaying hadron. This is $\mathcal{O}\left(m_{\mathrm{b}}\right)$ and $\mathcal{O}\left(m_{\mathrm{c}}\right)$ for B-decays and D-decays respectively. In the case of K-decays the typical choice is $\mu=\mathcal{O}(1-2 \mathrm{GeV})$ instead of $\mathcal{O}\left(m_{K}\right)$, which is much too low for any perturbative calculation of the couplings $C_{i}$.

Now due to the fact that $\mu \ll M_{W, Z}, m_{t}$, large logarithms $\ln M_{\mathrm{W}} / \mu$ compensate in the evaluation of $C_{i}(\mu)$ the smallness of the QCD coupling constant $\alpha_{s}$ and terms $\alpha_{s}^{n}\left(\ln M_{\mathrm{W}} / \mu\right)^{n}$, $\alpha_{s}^{n}\left(\ln M_{\mathrm{W}} / \mu\right)^{n-1}$ etc. have to be resummed to all orders in $\alpha_{s}$ before a reliable result for $C_{i}$ can be obtained. This can be done very efficiently by means of the renormalization group methods [13, 14, 15]. Indeed solving the renormalization group equations for the Wilson coefficients $C_{i}(\mu)$ summs automatically large logarithms. The resulting renormalization group improved perturbative expansion for $C_{i}(\mu)$ in terms of the effective coupling constant $\alpha_{s}(\mu)$ does not involve large logarithms and is more reliable.

It should be stressed at this point that the construction of the effective Hamiltonian $\mathcal{H}_{\text {eff }}$ by means of the operator product expansion and the renormalization group methods can be done fully in the perturbative framework. The fact that the decaying hadrons are bound states of quarks is irrelevant for this construction. Consequently the coefficients $C_{i}(\mu)$ are independent of the particular decay considered in the same manner in which the usual gauge couplings are universal and process independent.

Having constructed the effective Hamiltonian we can proceed to evaluate the decay amplitudes. An amplitude for a decay of a given meson $M=K, B,$. into a final state 
$F=\pi \nu \bar{\nu}, \pi \pi, D K$ is simply given by

$$
A(M \rightarrow F)=\left\langle F\left|\mathcal{H}_{e f f}\right| M\right\rangle=\frac{G_{F}}{\sqrt{2}} \sum_{i} V_{C K M}^{i} C_{i}(\mu)\left\langle F\left|Q_{i}(\mu)\right| M\right\rangle,
$$

where $\left\langle F\left|Q_{i}(\mu)\right| M\right\rangle$ are the hadronic matrix elements of $Q_{i}$ between $\mathrm{M}$ and $\mathrm{F}$. As indicated in (2.8) these matrix elements depend similarly to $C_{i}(\mu)$ on $\mu$. They summarize the physics contributions to the amplitude $A(M \rightarrow F)$ from scales lower than $\mu$.

We realize now the essential virtue of OPE: it allows to separate the problem of calculating the amplitude $A(M \rightarrow F)$ into two distinct parts: the short distance (perturbative) calculation of the couplings $C_{i}(\mu)$ and the long-distance (generally non-perturbative) calculation of the matrix elements $\left\langle Q_{i}(\mu)\right\rangle$. The scale $\mu$, as advertised above, separates then the physics contributions into short distance contributions contained in $C_{i}(\mu)$ and the long distance contributions contained in $\left\langle Q_{i}(\mu)\right\rangle$. By evolving this scale from $\mu=\mathcal{O}\left(M_{\mathrm{W}}\right)$ down to lower values one simply transforms the physics contributions at scales higher than $\mu$ from the hadronic matrix elements into $C_{i}(\mu)$. Since no information is lost this way the full amplitude cannot depend on $\mu$. Therefore the $\mu$-dependence of the couplings $C_{i}(\mu)$ has to cancel the $\mu$ dependence of $\left\langle Q_{i}(\mu)\right\rangle$. In other words it is a matter of choice what exactly belongs to $C_{i}(\mu)$ and what to $\left\langle Q_{i}(\mu)\right\rangle$. This cancellation of $\mu$-dependence involves generally several terms in the expansion in (2.8).

Clearly, in order to calculate the amplitude $A(M \rightarrow F)$, the matrix elements $\left\langle Q_{i}(\mu)\right\rangle$ have to be evaluated. Since they involve long distance contributions one is forced in this case to use non-perturbative methods such as lattice calculations, the $1 / \mathrm{N}$ expansion ( $\mathrm{N}$ is the number of colours), QCD sum rules, hadronic sum rules, chiral perturbation theory and so on. In the case of certain B-meson decays, the Heavy Quark Effective Theory (HQET) turns out to be a useful tool. Needless to say, all these non-perturbative methods have some limitations. Consequently the dominant theoretical uncertainties in the decay amplitudes reside in the matrix elements $\left\langle Q_{i}(\mu)\right\rangle$.

The fact that in most cases the matrix elements $\left\langle Q_{i}(\mu)\right\rangle$ cannot be reliably calculated at present, is very unfortunate. One of the main goals of the experimental studies of weak decays is the determination of the CKM factors $V_{\mathrm{CKM}}^{i}$ and the search for the physics beyond the Standard Model. Without a reliable estimate of $\left\langle Q_{i}(\mu)\right\rangle$ this goal cannot be achieved unless these matrix elements can be determined experimentally or removed from the final measurable quantities by taking the ratios or suitable combinations of amplitudes or branching ratios. However, this can be achieved only in a handful of decays and generally one has to face directly the calculation of $\left\langle Q_{i}(\mu)\right\rangle$.

Now in the case of semi-leptonic decays, in which there is at most one hadron in the final state, the chiral perturbation theory in the case of K-decays and HQET in the case of 
B-decays have already provided useful estimates of the relevant matrix elements. This way it was possible to achieve satisfactory determinations of the CKM elements $V_{u s}$ and $V_{c b}$ in $K \rightarrow \pi e \nu$ and $B \rightarrow D^{*} e \nu$ respectively. Similarly certain rare decays like $K \rightarrow \pi \nu \bar{\nu}$ and $B \rightarrow \mu \bar{\mu}$ can be calculated very reliably.

The case of non-leptonic decays in which the final state consists exclusively out of hadrons is a completely different story. Here even the matrix elements entering the simplest decays, the two-body decays like $K \rightarrow \pi \pi, D \rightarrow K \pi$ or $B \rightarrow D K$ cannot be calculated in QCD reliably at present. More promising in this respect is the evaluation of hadronic matrix elements relevant for $K^{0}-\bar{K}^{0}$ and $B_{d, s}^{0}-\bar{B}_{d, s}^{0}$ mixings.

Returning to the Wilson coefficients $C_{i}(\mu)$ it should be stressed that similar to the effective coupling constants they do not depend only on the scale $\mu$ but also on the renormalization scheme used: this time on the scheme for the renormalization of local operators. That the local operators undergo renormalization is not surprising. After all they represent effective vertices and as the usual vertices in a field theory they have to be renormalized when quantum corrections like QCD or QED corrections are taken into account. As a consequence of this, the hadronic matrix elements $\left\langle Q_{i}(\mu)\right\rangle$ are renormalization scheme dependent and this scheme dependence must be cancelled by the one of $C_{i}(\mu)$ so that the physical amplitudes are renormalization scheme independent. Again, as in the case of the $\mu$-dependence, the cancellation of the renormalization scheme dependence involves generally several terms in the expansion (2.8).

Now the $\mu$ and the renormalization scheme dependences of the couplings $C_{i}(\mu)$ can be evaluated efficiently in the renormalization group improved perturbation theory. Unfortunately the incorporation of these dependences in the non-perturbative evaluation of the matrix elements $\left\langle Q_{i}(\mu)\right\rangle$ remains as an important challenge and most of the non-perturbative methods on the market are insensitive to these dependences. The consequence of this unfortunate situation is obvious: the resulting decay amplitudes are $\mu$ and renormalization scheme dependent which introduces potential theoretical uncertainty in the predictions. On the other hand in certain decays these dependences can be put under control.

So far I have discussed only exclusive decays. It turns out that in the case of inclusive decays of heavy mesons, like B-mesons, things turn out to be easier. In an inclusive decay one sums over all (or over a special class) of accessible final states so that the amplitude for an inclusive decay takes the form:

$$
A(B \rightarrow X)=\frac{G_{F}}{\sqrt{2}} \sum_{f \in X} V_{\mathrm{CKM}}^{i} C_{i}(\mu)\left\langle f\left|Q_{i}(\mu)\right| B\right\rangle .
$$

At first sight things look as complicated as in the case of exclusive decays. It turns out, however, that the resulting branching ratio can be calculated in the expansion in inverse 
powers of $m_{\mathrm{b}}$ with the leading term described by the spectator model in which the B-meson decay is modelled by the decay of the $b$-quark:

$$
\operatorname{Br}(B \rightarrow X)=\operatorname{Br}(b \rightarrow q)+\mathcal{O}\left(\frac{1}{m_{\mathrm{b}}^{2}}\right) .
$$

This formula is known under the name of the Heavy Quark Expansion (HQE) [16]-18. Since the leading term in this expansion represents the decay of the quark, it can be calculated in perturbation theory or more correctly in the renormalization group improved perturbation theory. It should be realized that also here the basic starting point is the effective Hamiltonian (2.1) and that the knowledge of the couplings $C_{i}(\mu)$ is essential for the evaluation of the leading term in $(2.10)$. But there is an important difference relative to the exclusive case: the matrix elements of the operators $Q_{i}$ can be "effectively" evaluated in perturbation theory. This means, in particular, that their $\mu$ and renormalization scheme dependences can be evaluated and the cancellation of these dependences by those present in $C_{i}(\mu)$ can be explicitly investigated.

Clearly in order to complete the evaluation of $\operatorname{Br}(B \rightarrow X)$ also the remaining terms in (2.10) have to be considered. These terms are of a non-perturbative origin, but fortunately they are suppressed by at least two powers of $m_{b}$. They have been studied by several authors in the literature with the result that they affect various branching ratios by less than $10 \%$ and often by only a few percent. Consequently the inclusive decays give generally more precise theoretical predictions at present than the exclusive decays. On the other hand their measurements are harder. There is of course an important theoretical issue related to the validity of HQE in (2.10) which appear in the literature under the name of quark-hadron duality. I will not discuss it here. Recent discussions of this issue can be found in [19].

We have learned now that the matrix elements of $Q_{i}$ are easier to handle in inclusive decays than in the exclusive ones. On the other hand the evaluation of the couplings $C_{i}(\mu)$ is equally difficult in both cases although as stated above it can be done in a perturbative framework. Still in order to achieve sufficient precision for the theoretical predictions it is desirable to have accurate values of these couplings. Indeed it has been realized at the end of the eighties that the leading term (LO) in the renormalization group improved perturbation theory, in which the terms $\alpha_{s}^{n}\left(\ln M_{\mathrm{W}} / \mu\right)^{n}$ are summed, is generally insufficient and the inclusion of next-to-leading corrections (NLO) which correspond to summing the terms $\alpha_{s}^{n}\left(\ln M_{\mathrm{W}} / \mu\right)^{n-1}$ is necessary. In particular, unphysical left-over $\mu$-dependences in the decay amplitudes and branching ratios resulting from the truncation of the perturbative series are considerably reduced by including NLO corrections. These corrections are known by now for the most important and interesting decays and will be briefly reviewed below. 


\section{Penguin-Box Expansion and OPE}

The FCNC decays, in particular rare and CP violating decays are governed by various penguin and box diagrams with internal top quark and charm quark exchanges. Some examples are shown in fig. 2. These diagrams can be evaluated in the full theory and are summarized by a set of basic universal (process independent) $m_{\mathrm{t}}$-dependent functions $F_{r}\left(x_{t}\right)$ [20] where $x_{t}=m_{\mathrm{t}}^{2} / M_{\mathrm{W}}^{2}$. Explicit expressions for these functions can be found in [21, 22, 23].

It is useful to express the OPE formula (2.8) directly in terms of the functions $F_{r}\left(x_{t}\right)$ [25]. To this end we rewrite the $A(M \rightarrow F)$ in $(2.8)$ as follows

$$
A(M \rightarrow F)=\frac{G_{\mathrm{F}}}{\sqrt{2}} V_{\mathrm{CKM}} \sum_{i, k}\left\langle F\left|O_{k}(\mu)\right| M\right\rangle \hat{U}_{k i}\left(\mu, M_{\mathrm{W}}\right) C_{i}\left(M_{\mathrm{W}}\right),
$$

where $\hat{U}_{k j}\left(\mu, M_{W}\right)$ is the renormalization group transformation from $M_{\mathrm{W}}$ down to $\mu$. Explicit formula for this transformation will be given below. In order to simplify the presentation we have removed the index "i" from $V_{\text {CKM }}^{i}$

Now $C_{i}\left(M_{\mathrm{W}}\right)$ are linear combinations of the basic functions $F_{r}\left(x_{t}\right)$ so that we can write

$$
C_{i}\left(M_{\mathrm{W}}\right)=c_{i}+\sum_{r} h_{i r} F_{r}\left(x_{t}\right)
$$

where $c_{i}$ and $h_{i r}$ are $m_{\mathrm{t}}$-independent constants. Inserting (3.12) into (3.11) and summing over $i$ and $k$ we find

$$
A(M \rightarrow F)=P_{0}(M \rightarrow F)+\sum_{r} P_{r}(M \rightarrow F) F_{r}\left(x_{t}\right)
$$

with

$$
\begin{gathered}
P_{0}(M \rightarrow F)=\sum_{i, k}\left\langle F\left|O_{k}(\mu)\right| M\right\rangle \hat{U}_{k i}\left(\mu, M_{\mathrm{W}}\right) c_{i}, \\
P_{r}(M \rightarrow F)=\sum_{i, k}\left\langle F\left|O_{k}(\mu)\right| M\right\rangle \hat{U}_{k i}\left(\mu, M_{\mathrm{W}}\right) h_{i r},
\end{gathered}
$$

where we have suppressed the overall factor $\left(G_{F} / \sqrt{2}\right) V_{C K M}$. I would like to call (3.13) Penguin-Box Expansion (PBE) [25].

The coefficients $P_{0}$ and $P_{r}$ are process dependent. This process dependence enters through $\left\langle F\left|O_{k}(\mu)\right| M\right\rangle$. In certain cases like $K \rightarrow \pi \nu \bar{\nu}$ these matrix elements are very simple implying simple formulae for the coefficients $P_{0}$ and $P_{r}$. In other situations, like $\varepsilon^{\prime} / \varepsilon$, this is not the case.

Originally PBE was designed to expose the $m_{\mathrm{t}}$-dependence of FCNC processes [25]. After the top quark mass has been measured precisely this role of PBE is less important. On the other hand, PBE is very well suited for the study of the extentions of the Standard Model in which new particles are exchanged in the loops. We know already that these particles are 
heavier than W-bosons and consequently they can be integrated out together with the weak bosons and the top quark. If there are no new local operators the mere change is to modify the functions $F_{r}\left(x_{t}\right)$ which now acquire the dependence on the masses of new particles such as charged Higgs particles and supersymmetric particles. The process dependent coefficients $P_{0}$ and $P_{r}$ remain unchanged. This is particularly useful as the most difficult part is the evaluation of $\hat{U}_{k j}\left(\mu, M_{W}\right)$ and of the hadronic matrix elements, both contained in these coefficients. However, if new effective operators with different Dirac and colour structures are present the values of $P_{0}$ and $P_{r}$ are modified. Examples of the applications of PBE to physics beyond the Standard Model can be found in [26, 27, 28].

The universality of the functions $F_{r}\left(x_{t}\right)$ can be violated partly when QCD corrections to one loop penguin and box diagrams are included. For instance in the case of semi-leptonic FCNC transitions there is no gluon exchange in a $Z^{0}$-penguin diagram parallel to the $Z^{0}$ propagator but such an exchange takes place in non-leptonic decays in which the bottom line is a quark-line. Thus the general universality of $F_{r}\left(x_{t}\right)$ present at one loop level is reduced to two universality classes relevant for semi-leptonic and non-leptonic transitions. However, the $\mathcal{O}\left(\alpha_{s}\right)$ corrections to the functions $F_{r}\left(x_{t}\right)$ are generally rather small when the top quark mass $\bar{m}_{\mathrm{t}}\left(m_{\mathrm{t}}\right)$ is used and consequently the inclusion of QCD effects plays mainly the role in reducing various $\mu$-dependences.

In order to see the general structure of $A(M \rightarrow F)$ more transparently let us write it as follows:

$$
A(M \rightarrow F)=B_{M \rightarrow F} V_{\mathrm{CKM}} \eta_{\mathrm{QCD}} F\left(x_{t}\right)+\text { Charm }
$$

where the first term represents the internal top quark contribution and "Charm" stands for remaining contributions, in particular those with internal charm quark exchanges. $F\left(x_{t}\right)$ represents one of the universal functions and $\eta_{\mathrm{QCD}}$ the corresponding short distance QCD corrections. The parameter $B_{M \rightarrow F}$ represents the relevant hadronic matrix element, which can only be calculated by means of non-perturbative methods. However, in certain lucky situations $B_{M \rightarrow F}$ can be extracted from well measured leading decays and when it enters also other decays, the latter are then free from hadronic uncertainties and offer very useful means for extraction of CKM parameters. One such example is the decay $K^{+} \rightarrow \pi^{+} \nu \bar{\nu}$ for which one has

$$
B r\left(K^{+} \rightarrow \pi^{+} \nu \bar{\nu}\right)=\left[\frac{\alpha_{\mathrm{QED}}^{2} B r\left(K^{+} \rightarrow \pi^{0} e^{+} \nu\right)}{V_{u s}^{2} 2 \pi^{2} \sin ^{4} \theta_{W}}\right] \cdot\left|V_{t s}^{*} V_{t d} \eta_{\mathrm{QCD}}^{t} F\left(x_{t}\right)+V_{c s}^{*} V_{c d} \eta_{\mathrm{QCD}}^{c} F\left(x_{c}\right)\right|^{2}
$$

The factor in square brackets stands for the " $B$ - factor" in (3.16), which is given in terms of well measured quantities. Since $V_{c s}, V_{c d}$ and $V_{t s}$ are already rather well determined and 
$F\left(x_{i}\right)$ and $\eta_{\mathrm{QCD}}^{i}$ can be calculated in perturbation theory, the element $V_{t d}$ can be extracted from $\operatorname{Br}\left(K^{+} \rightarrow \pi^{+} \nu \bar{\nu}\right)$ without essentially any theoretical uncertainties. We will be more specific about this in Section 7.

\section{Motivations for NLO Calculations}

Going beyond the LO approximation for $C_{i}(\mu)$ is certainly an important but a non-trivial step. For this reason one needs some motivations to perform this step. Here are the main reasons for going beyond LO:

- The NLO is first of all necessary to test the validity of the renormalization group improved perturbation theory.

- Without going to NLO the QCD scale $\Lambda_{\overline{M S}}$ [29] extracted from various high energy processes cannot be used meaningfully in weak decays.

- Due to renormalization group invariance the physical amplitudes do not depend on the scales $\mu$ present in $\alpha_{s}$ or in the running quark masses, in particular $m_{\mathrm{t}}(\mu), m_{\mathrm{b}}(\mu)$ and $m_{\mathrm{c}}(\mu)$. However, in perturbation theory this property is broken through the truncation of the perturbative series. Consequently one finds sizable scale ambiguities in the leading order, which can be reduced considerably by going to NLO.

- The Wilson Coefficients are renormalization scheme dependent quantities. This scheme dependence appears first at NLO. For a proper matching of the short distance contributions to the long distance matrix elements obtained from lattice calculations it is essential to calculate NLO. The same is true for inclusive heavy quark decays in which the hadron decay can be modeled by a decay of a heavy quark and the matrix elements of $Q_{i}$ can be effectively calculated in an expansion in $1 / m_{\mathrm{b}}$.

- In several cases the central issue of the top quark mass dependence is strictly a NLO effect.

\section{General Structure of Wilson Coefficients}

We will give here a formula for the Wilson coefficient $C(\mu)$ of a single operator $Q$ including NLO corrections. The case of several operators which mix under renormalization is much more complicated. Explicit formulae are given in 21, 23].

$C(\mu)$ is given by

$$
C(\mu)=U\left(\mu, M_{\mathrm{W}}\right) C\left(M_{\mathrm{W}}\right)
$$


where

$$
U\left(\mu, M_{\mathrm{W}}\right)=\exp \left[\int_{g_{s}\left(M_{\mathrm{W}}\right)}^{g_{s}(\mu)} d g^{\prime} \frac{\gamma_{Q}\left(g_{s}^{\prime}\right)}{\beta\left(g_{s}^{\prime}\right)}\right]
$$

is the evolution function, which allows to calculate $C(\mu)$ once $C\left(M_{\mathrm{W}}\right)$ is known. The latter can be calculated in perturbation theory in the process of integrating out $W^{ \pm}, Z^{0}$ and top quark fields. Details can be found in [21, 23]. Next $\gamma_{Q}$ is the anomalous dimension of the operator $\mathrm{Q}$ and $\beta\left(g_{s}\right)$ is the renormalization group function which governs the evolution of the QCD coupling constant $\alpha_{s}(\mu)$.

At NLO we have

$$
\begin{gathered}
C\left(M_{W}\right)=1+\frac{\alpha_{s}\left(M_{W}\right)}{4 \pi} B \\
\gamma_{Q}\left(\alpha_{s}\right)=\gamma_{Q}^{(0)} \frac{\alpha_{s}}{4 \pi}+\gamma_{Q}^{(1)}\left(\frac{\alpha_{s}}{4 \pi}\right)^{2} \\
\beta\left(g_{s}\right)=-\beta_{0} \frac{g_{s}^{3}}{16 \pi^{2}}-\beta_{1} \frac{g_{s}^{5}}{\left(16 \pi^{2}\right)^{2}}
\end{gathered}
$$

Inserting the last two formulae into (5.19) and expanding in $\alpha_{s}$ we find

$$
U\left(\mu, M_{\mathrm{W}}\right)=\left[1+\frac{\alpha_{s}(\mu)}{4 \pi} J\right]\left[\frac{\alpha_{s}\left(M_{W}\right)}{\alpha_{s}(\mu)}\right]^{d}\left[1-\frac{\alpha_{s}\left(M_{W}\right)}{4 \pi} J\right]
$$

with

$$
J=\frac{d}{\beta_{0}} \beta_{1}-\frac{\gamma_{Q}^{(1)}}{2 \beta_{0}} \quad d=\frac{\gamma_{Q}^{(0)}}{2 \beta_{0}} .
$$

Inserting (5.23) and (5.20) into (5.18) we find an important formula for $C(\mu)$ in the NLO approximation:

$$
C(\mu)=\left[1+\frac{\alpha_{s}(\mu)}{4 \pi} J\right]\left[\frac{\alpha_{s}\left(M_{W}\right)}{\alpha_{s}(\mu)}\right]^{d}\left[1+\frac{\alpha_{s}\left(M_{W}\right)}{4 \pi}(B-J)\right] .
$$

\section{Status of NLO Calculations}

Since the pioneering leading order calculations of Wilson coefficients for current-current [5, 6] and penguin operators [30], enormous progress has been made, so that at present most of the decay amplitudes are known at the NLO level. We list all existing NLO calculations for weak decays in table 1. In addition to the calculations in the Standard Model we list the calculations in two-Higgs doublet models and supersymmetry. In table 2 we list references to calculations of two-loop electroweak contributions to rare decays. The latter calculations allow to reduce scheme and scale dependences related to the definition of electroweak parameters like $\sin ^{2} \theta_{W}$, $\alpha_{Q E D}$, etc. Next, useful techniques for three-loop calculations can be found in 77 and a very 
general discussion of the evanescent operators including earlier references is presented in [78]. Further details on these calculations can be found in the orignal papers, in the review 21] and in the Les Houches lectures [23]. Some of the implications of these calculations will be analyzed briefly in subsequent sections.

\section{Applications: News}

\subsection{Preliminaries}

There is a vast literature on the applications of NLO calculations listed in table 1. As they are already reviewed in detail in [21, 22, 23] there is no point to review them here again. I will rather discuss briefly some of the most important applications in general terms. This will also give me the opportunity to update some of the numerical results presented in [23]. This update is related mainly to the improved experimental lower bound on $B_{s}^{0}-\bar{B}_{s}^{0}$ mixing $\left((\Delta M)_{s}>12.4 / p s\right)$ and a slight increase in $\left|V_{u b}\right| /\left|V_{c b}\right|: 0.091 \pm 0.016$, both presented at the last Rochester Conference in Vancouver [79].

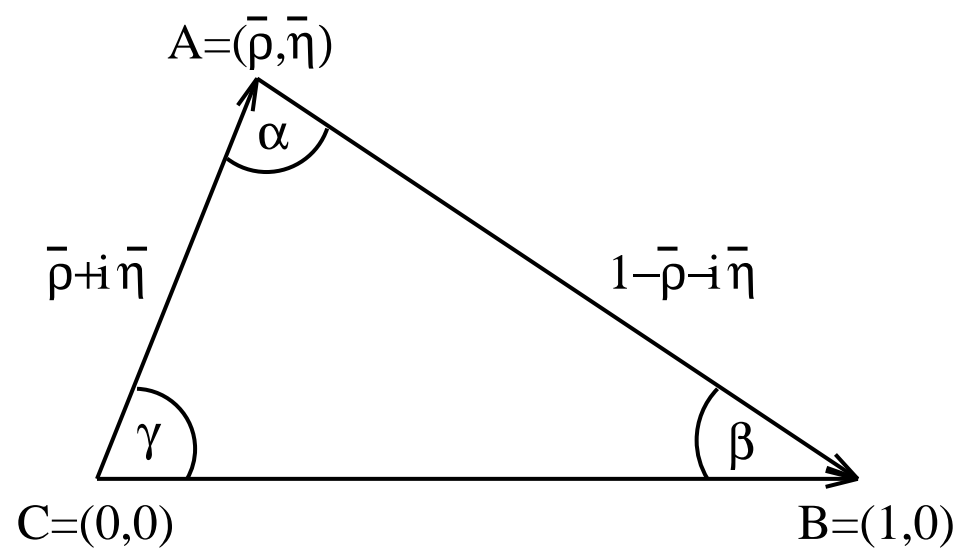

Figure 3: Unitarity Triangle.

\section{$7.2 \quad$ Unitarity Triangle}

The standard analysis of the unitarity triangle (see fig. 3) uses the values of $\left|V_{u s}\right|,\left|V_{c b}\right|$, $\left|V_{u b} / V_{c b}\right|$ extracted from tree level K- and B- decays, the indirect CP-violation in $K_{L} \rightarrow \pi \pi$ represented by the parameter $\varepsilon$ and the $B_{d, s}^{0}-\bar{B}_{d, s}^{0}$ mixings described by the mass differences $(\Delta M)_{d, s}$. From this analysis follows the allowed range for $(\bar{\varrho}, \bar{\eta})$ describing the apex of the unitarity triangle. Here 81

$$
\bar{\varrho}=\varrho\left(1-\frac{\lambda^{2}}{2}\right), \quad \bar{\eta}=\eta\left(1-\frac{\lambda^{2}}{2}\right) .
$$


where $\lambda, \varrho$ and $\eta$ are Wolfenstein parameters [82] with $\left|V_{u s}\right|=\lambda=0.22$. We have in particular

$$
V_{u b}=\lambda\left|V_{c b}\right|(\varrho-i \eta), \quad V_{t d}=\lambda\left|V_{c b}\right|(1-\bar{\varrho}-i \bar{\eta})
$$

$\eta \neq 0$ is responsible for $\mathrm{CP}$ violation in the Standard Model.

The allowed region for $(\bar{\varrho}, \bar{\eta})$ is presented in fig. 田. It is the shaded area on the right hand side of the solid circle which represents the upper bound for $(\Delta M)_{d} /(\Delta M)_{s}$. The hyperbolas give the constraint from $\varepsilon$ and the two circles centered at $(0,0)$ the constraint from $\left|V_{u b} / V_{c b}\right|$. The white areas between the lower $\varepsilon$-hyperbola and the shaded region are excluded by $B_{d}^{0}-\bar{B}_{d}^{0}$ mixing. We observe that the region $\bar{\varrho}<0$ is practically excluded. The main remaining theoretical uncertainties in this analysis are the values of non-perturbative parameters: $B_{K}$ in $\varepsilon, F_{B_{d}} \sqrt{B_{d}}$ in $(\Delta M)_{d}$ and $\xi=F_{B_{s}} \sqrt{B_{s}} / F_{B_{d}} \sqrt{B_{d}}$ in $(\Delta M)_{d} /(\Delta M)_{s}$. I have used $B_{K}=0.80 \pm 0.15, F_{B_{d}} \sqrt{B_{d}}=200 \pm 40 \mathrm{MeV}$ and $\xi_{\max }=1.2$. On the experimental side $\left|V_{u b} / V_{c b}\right|$ and $(\Delta M)_{s}$ should be improved.

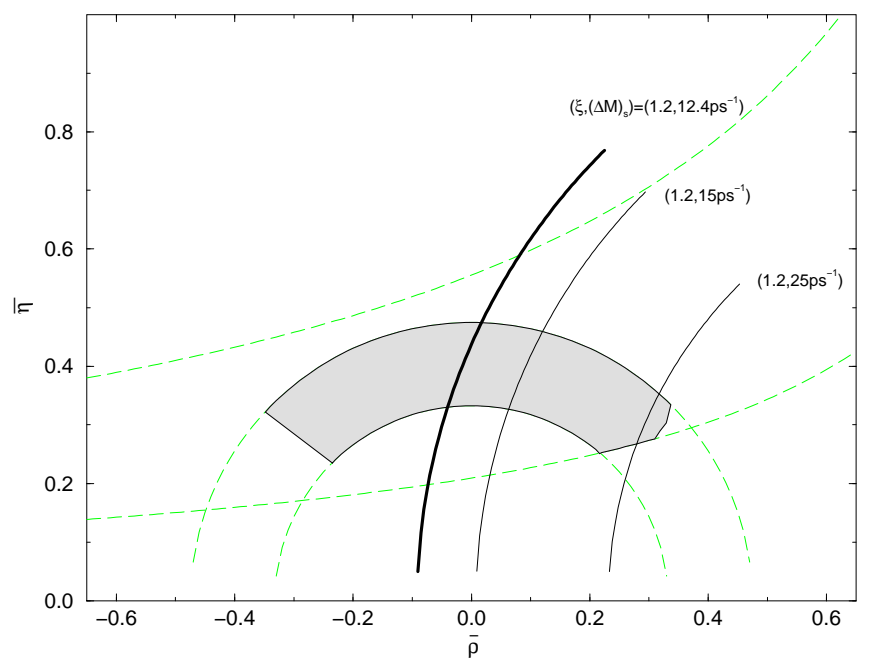

Figure 4: Unitarity Triangle 1998.

From this analysis we extract

$$
\left|V_{t d}\right|=(8.6 \pm 1.6) \cdot 10^{-3}, \quad \operatorname{Im}\left(V_{t s}^{*} V_{t d}\right)=(1.38 \pm 0.33) \cdot 10^{-4}
$$

and

$$
\sin 2 \beta=0.71 \pm 0.13, \quad \sin \gamma=0.83 \pm 0.17
$$

\section{3 $\varepsilon^{\prime} / \varepsilon$}

$\varepsilon^{\prime} / \varepsilon$ is the ratio of the direct and indirect $\mathrm{CP}$ violation in $K_{L} \rightarrow \pi \pi$. A measurement of a non-vanishing value of $\varepsilon^{\prime} / \varepsilon$ would give the first signal for the direct $\mathrm{CP}$ violation ruling 
out the superweak models [83]. In the Standard Model $\varepsilon^{\prime} / \varepsilon$ is governed by QCD penguins and electroweak (EW) penguins. The corresponding operators are given in (2.4)-(2.7). With increasing $m_{\mathrm{t}}$ the EW penguins become increasingly important 84, 85], and entering $\varepsilon^{\prime} / \varepsilon$ with the opposite sign to QCD penguins suppress this ratio for large $m_{\mathrm{t}}$. For $m_{\mathrm{t}} \approx 200 \mathrm{GeV}$ the ratio can even be zero 85]. Because of this strong cancellation between two dominant contributions and due to uncertainties related to hadronic matrix elements of the relevant local operators, a precise prediction of $\varepsilon^{\prime} / \varepsilon$ is not possible at present.

A very simplified formula (not to be used for any serious numerical analysis) which exhibits main uncertainties is given as follows

$$
\frac{\varepsilon^{\prime}}{\varepsilon}=15 \cdot 10^{-4}\left[\frac{\eta \lambda\left|V_{c b}\right|^{2}}{1.3 \cdot 10^{-4}}\right]\left[\frac{120 \mathrm{MeV}}{m_{s}(2 \mathrm{GeV})}\right]^{2}\left[\frac{\Lambda_{\frac{\mathrm{MS}}{(4)}}}{300 \mathrm{MeV}}\right]^{0.8}\left[B_{6}-Z\left(x_{t}\right) B_{8}\right]
$$

where $Z\left(x_{t}\right) \approx 0.18\left(m_{\mathrm{t}} / M_{\mathrm{W}}\right)^{1.86}$ represents the leading $m_{\mathrm{t}}$-dependence of EW penguins. $B_{6}$ and $B_{8}$ represent the hadronic matrix elements of the dominant QCD-penguin operator $Q_{6}$ and the dominant electroweak penguin operator $Q_{8}$ (see (2.5) and (2.6) respectively. Together with $m_{\mathrm{s}}(2 \mathrm{GeV})$ the values of these parameters constitute the main theoretical uncertainty in evaluating $\varepsilon^{\prime} / \varepsilon$. Present status of $m_{\mathrm{s}}, B_{6}$ and $B_{8}$ is reviewed in [86, 23]. Roughly one has $B_{6}=1.0 \pm 0.2$ and $B_{8}=0.7 \pm 0.2$. Taking these values, $\eta$ of fig. 1 and $\left|V_{c b}\right|=0.040 \pm 0.003$, I find:

$$
\varepsilon^{\prime} / \varepsilon= \begin{cases}(5.7 \pm 3.6) \cdot 10^{-4}, & m_{\mathrm{s}}(2 \mathrm{GeV})=130 \pm 20 \mathrm{MeV} \\ (9.1 \pm 5.7) \cdot 10^{-4}, & m_{\mathrm{s}}(2 \mathrm{GeV})=110 \pm 20 \mathrm{MeV}\end{cases}
$$

where the chosen values for $m_{\mathrm{s}}$ are in the ball park of various QCD sum rules and lattice estimates 86]. This should be compared with the result of NA31 collaboration at CERN which finds $\left(\varepsilon^{\prime} / \varepsilon\right)=(23 \pm 7) \cdot 10^{-4}$ [87] and the value of E731 at Fermilab, $\left(\varepsilon^{\prime} / \varepsilon\right)=(7.4 \pm$ $5.9) \cdot 10^{-4} 88$.

The Standard Model expectations are closer to the Fermilab result, but due to large theoretical and experimental errors no firm conclusion can be reached at present. The new improved data from CERN and Fermilab in 1999 and later from DAФNE should shed more light on $\varepsilon^{\prime} / \varepsilon$. In this context improved estimates of $B_{6}, B_{8}$ and $m_{\mathrm{s}}$ are clearly desirable.

\section{$7.4 \quad B \rightarrow X_{s} \gamma$}

A lot of efforts have been put into predicting the branching ratio for the inclusive decay $B \rightarrow X_{s} \gamma$ including NLO QCD corrections and higher order electroweak corrections. The relevant references are given in table 1 and in [23], where details can be found. The final 
result of these efforts can be summarized by

$$
\operatorname{Br}\left(B \rightarrow X_{s} \gamma\right)_{\mathrm{th}}=(3.30 \pm 0.15(\text { scale }) \pm 0.26(\text { par })) \cdot 10^{-4}
$$

where the first error represents residual scale dependences and the second error is due to uncertainties in input parameters. The main achievement is the reduction of the scale dependence through NLO calculations, in particular those given in 61] and 40. In the leading order the corresponding error would be roughly \pm 0.6 [89, 90].

The theoretical result in (7.32) should be compared with experimental data:

$$
B r\left(B \rightarrow X_{s} \gamma\right)_{\exp }= \begin{cases}(3.15 \pm 0.35 \pm 0.41) \cdot 10^{-4}, & \text { CLEO } \\ (3.11 \pm 0.80 \pm 0.72) \cdot 10^{-4}, & \text { ALEPH }\end{cases}
$$

which implies the combined branching ratio:

$$
\operatorname{Br}\left(B \rightarrow X_{s} \gamma\right)_{\exp }=(3.14 \pm 0.48) \cdot 10^{-4}
$$

Clearly, the Standard Model result agrees well with the data. In order to see whether any new physics can be seen in this decay, the theoretical and in particular experimental errors should be reduced. This is certainly a very difficult task. Most recent analyses of $B \rightarrow X_{s} \gamma$ in supersymmetric models and two-Higgs doublet models are listed in table 1 .

\section{5 $\quad K_{L} \rightarrow \pi^{0} \nu \bar{\nu}$ and $K^{+} \rightarrow \pi^{+} \nu \bar{\nu}$}

$K_{L} \rightarrow \pi^{0} \nu \bar{\nu}$ and $K^{+} \rightarrow \pi^{+} \nu \bar{\nu}$ are the theoretically cleanest decays in the field of rare $\mathrm{K}$ decays. $K_{L} \rightarrow \pi^{0} \nu \bar{\nu}$ is dominated by short distance loop diagrams (Z-penguins and box diagrams) involving the top quark. $K^{+} \rightarrow \pi^{+} \nu \bar{\nu}$ receives additional sizable contributions from internal charm exchanges. The great virtue of $K_{L} \rightarrow \pi^{0} \nu \bar{\nu}$ is that it proceeds almost exclusively through direct CP violation [91 and as such is the cleanest decay to measure this important phenomenon. It also offers a clean determination of the Wolfenstein parameter $\eta$ and in particular offers the cleanest measurement of $\operatorname{Im} V_{t s}^{*} V_{t d}$ 92]. $K^{+} \rightarrow \pi^{+} \nu \bar{\nu}$ is CP conserving and offers a clean determination of $\left|V_{t d}\right|$. Due to the presence of the charm contribution and the related $m_{c}$ dependence it has a small scale uncertainty absent in $K_{L} \rightarrow$ $\pi^{0} \nu \bar{\nu}$.

The next-to-leading QCD corrections [49, 50, 53, 51, 52 to both decays considerably reduced the theoretical uncertainty due to the choice of the renormalization scales present in the leading order expressions, in particular in the charm contribution to $K^{+} \rightarrow \pi^{+} \nu \bar{\nu}$. Since the relevant hadronic matrix elements of the weak currents entering $K \rightarrow \pi \nu \bar{\nu}$ can be related using isospin symmetry to the leading decay $K^{+} \rightarrow \pi^{0} e^{+} \nu$, the resulting theoretical expressions for $\operatorname{Br}\left(K_{L} \rightarrow \pi^{0} \nu \bar{\nu}\right)$ and $\operatorname{Br}\left(K^{+} \rightarrow \pi^{+} \nu \bar{\nu}\right)$ are only functions of the CKM 
parameters, the QCD scale $\Lambda_{\overline{\mathrm{MS}}}$ and the quark masses $m_{\mathrm{t}}$ and $m_{\mathrm{c}}$. The isospin braking corrections have been calculated in [93]. The long distance contributions to $K^{+} \rightarrow \pi^{+} \nu \bar{\nu}$ have been considered in [94] and found to be very small: a few percent of the charm contribution to the amplitude at most, which is safely neglegible. The long distance contributions to $K_{L} \rightarrow \pi^{0} \nu \bar{\nu}$ are negligible as well [95].

The explicit expressions for $\operatorname{Br}\left(K^{+} \rightarrow \pi^{+} \nu \bar{\nu}\right)$ and $\operatorname{Br}\left(K_{L} \rightarrow \pi^{0} \nu \bar{\nu}\right)$ can be found in [21, 22, 23]. Here we give approximate expressions in order to exhibit various dependences:

$$
\begin{gathered}
B r\left(K^{+} \rightarrow \pi^{+} \nu \bar{\nu}\right)=0.7 \cdot 10^{-10}\left[\left[\frac{\left|V_{t d}\right|}{0.010}\right]^{2}\left[\frac{\left|V_{c b}\right|}{0.040}\right]^{2}\left[\frac{\bar{m}_{\mathrm{t}}\left(m_{\mathrm{t}}\right)}{170 \mathrm{GeV}}\right]^{2.3}+\mathrm{cc}+\mathrm{tc}\right] \\
\operatorname{Br}\left(K_{L} \rightarrow \pi^{0} \nu \bar{\nu}\right)=3.0 \cdot 10^{-11}\left[\frac{\eta}{0.39}\right]^{2}\left[\frac{\bar{m}_{\mathrm{t}}\left(m_{\mathrm{t}}\right)}{170 \mathrm{GeV}}\right]^{2.3}\left[\frac{\left|V_{c b}\right|}{0.040}\right]^{4}
\end{gathered}
$$

where in (7.35) we have shown explicitly only the pure top contribution.

The impact of NLO calculations is the reduction of scale uncertainties in $\operatorname{Br}\left(K^{+} \rightarrow \pi^{+} \nu \bar{\nu}\right)$ from $\pm 23 \%$ to $\pm 7 \%$. This corresponds to the reduction in the uncertainty in the determination of $\left|V_{t d}\right|$ from $\pm 14 \%$ to $\pm 4 \%$. The remaining scale uncertainties in $\operatorname{Br}\left(K_{L} \rightarrow \pi^{0} \nu \bar{\nu}\right)$ and in the determination of $\eta$ are fully negligible.

Updating the analysis of [23] one finds [52]:

$$
\operatorname{Br}\left(K^{+} \rightarrow \pi^{+} \nu \bar{\nu}\right)=(8.2 \pm 3.2) \cdot 10^{-11} \quad, \quad \operatorname{Br}\left(K_{L} \rightarrow \pi^{0} \nu \bar{\nu}\right)=(3.1 \pm 1.3) \cdot 10^{-11}
$$

where the errors come dominantly from the uncertainties in the CKM parameters.

As stressed in [92] simultaneous measurements of $\operatorname{Br}\left(K^{+} \rightarrow \pi^{+} \nu \bar{\nu}\right)$ and $\operatorname{Br}\left(K_{L} \rightarrow \pi^{0} \nu \bar{\nu}\right)$ should allow a clean determination of the unitarity triangle as shown in fig. 5 . In particular the measurements of these branching ratios with an error of $\pm 10 \%$ will determine $\left|V_{t d}\right|$, $\operatorname{Im} V_{t s}^{*} V_{t d}$ and $\sin 2 \beta$ with an accuraccy of $\pm 10 \%, \pm 5 \%$ and \pm 0.05 respectively. The comparision of this determination of $\sin 2 \beta$ with the one by means of the CP-asymmetry in $B \rightarrow \psi K_{S}$ should offer a very good test of the Standard Model.

Experimentally we have [96]

$$
B R\left(K^{+} \rightarrow \pi^{+} \nu \bar{\nu}\right)=(4.2+9.7-3.5) \cdot 10^{-10}
$$

and the bound [97]

$$
B R\left(K_{\mathrm{L}} \rightarrow \pi^{0} \nu \bar{\nu}\right)<1.6 \cdot 10^{-6}
$$

Moreover from (7.38) and isospin symmetry one has [98] $B R\left(K_{\mathrm{L}} \rightarrow \pi^{0} \nu \bar{\nu}\right)<6.1 \cdot 10^{-9}$.

The central value in (7.38) is by a factor of 4 above the Standard Model expectation but in view of large errors the result is compatible with the Standard Model. The analysis 


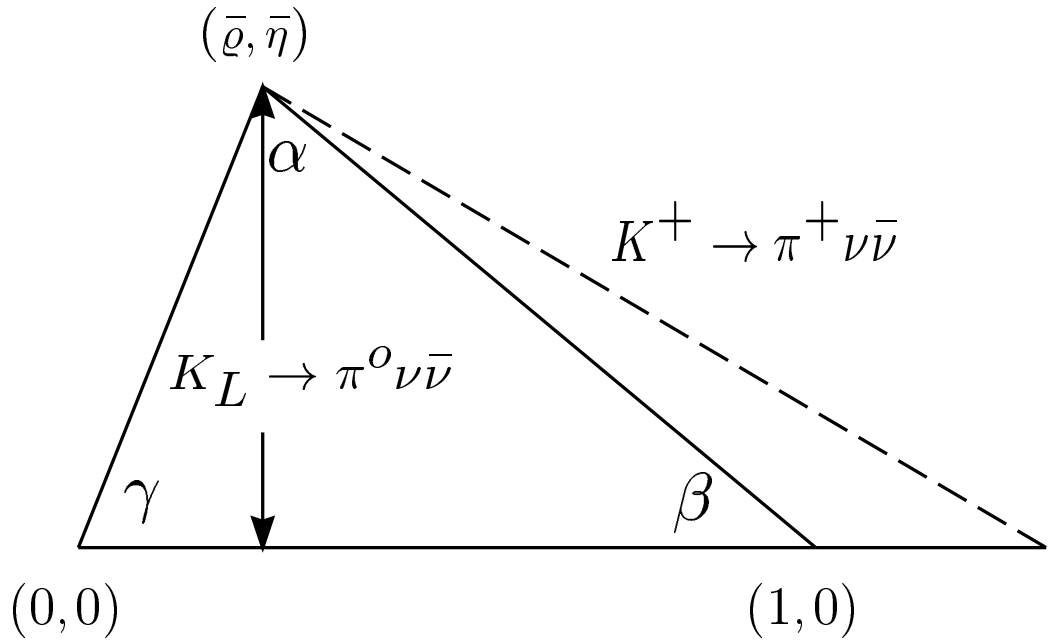

Figure 5: Unitarity triangle from $K \rightarrow \pi \nu \bar{\nu}$.

of additional data on $K^{+} \rightarrow \pi^{+} \nu \bar{\nu}$ present on tape at BNL787 should narrow this range in the near future considerably. In view of the clean character of this decay a measurement of its branching ratio at the level of $2 \cdot 10^{-10}$ would signal the presence of physics beyond the Standard Model [52]. The Standard Model sensitivity is expected to be reached at AGS around the year 2000 [99]. Also Fermilab with the Main Injector could measure this decay [100].

The present upper bound on $\operatorname{Br}\left(K_{\mathrm{L}} \rightarrow \pi^{0} \nu \bar{\nu}\right)$ is about five orders of magnitude above the Standard Model expectation (7.37). FNAL-E799 expects to reach the accuracy $\mathcal{O}\left(10^{-8}\right)$ and a very interesting new experiment at Brookhaven (BNL E926) [99] expects to reach the single event sensitivity $2 \cdot 10^{-12}$ allowing a $10 \%$ measurement of the expected branching ratio. There are furthermore plans to measure this gold-plated decay with comparable sensitivity at Fermilab [101] and KEK [102].

\section{Summary}

We have given a general description of OPE and Renormalization Group techniques as applied to weak decays of mesons. Further details can be found in [21, 22, 23, 103]. One of the outstanding and important challanges for theorists in this field is a quantitative description of non-leptonic meson decays. In the field of $\mathrm{K}$-decays this is in particular the case of the $\Delta I=1 / 2$ rule for which some progress has been made in [104. In the field of $\mathrm{B}$ decays progress in a quantitative description of two-body decays is very desirable in view of forthcoming B-physics experiments at Cornell, SLAC, KEK, DESY, FNAL and later at LHC. 
Recent reviews on non-leptonic two-body decays are given in 105, 106, 107 where further references can be found.

I would like to thank Peter Breitenlohner, Dieter Maison and Julius Wess for inviting me to such a pleasant symposium.

\section{References}

[1] K.G. Wilson, Phys. Rev. 179 (1969) 1499;

[2] W. Zimmermann, in Proc. 1970 Brandeis Summer Institute in Theor. Phys, (eds. S. Deser, M. Grisaru and H. Pendleton), MIT Press, 1971, p.396;

[3] K.G. Wilson and W. Zimmermann, Comm. Math. Phys. 24 (1972) 87.

[4] W. Zimmermann, Ann. Phys. 77 (1973) 570.

[5] M.K. Gaillard and B.W. Lee, Phys. Rev. Lett. 33 (1974) 108.

[6] G. Altarelli and L. Maiani, Phys. Lett. B 52 (1974) 351.

[7] N. Cabibbo, Phys. Rev. Lett. 10 (1963) 531.

[8] M. Kobayashi and K. Maskawa, Prog. Theor. Phys. 49 (1973) 652.

[9] E.C.G. Sudarshan and R.E. Marshak, Proc. Padua-Venice Conf. on Mesons and Recently Discovered Particles (1957).

[10] R.P. Feynman and M. Gell-Mann, Phys. Rev. 109 (1958) 193.

[11] D. Zeppenfeld, Z. Phys. C 8 (1981) 77; L.L. Chau, Phys. Rev. D 43 (1991) 2176; M. Gronau, J.L. Rosner and D. London, Phys. Rev. Lett. 73 (1994) 21; O.F. Hernandez, M. Gronau, J.L. Rosner and D. London, Phys. Lett. B 333 (1994) 500, Phys. Rev. D 50 (1994) 4529.

[12] E. Witten, Nucl. Phys. B 120 (1977) 189.

[13] E.C.G. Stueckelberg and A. Petermann, Helv. Phys. Acta 26 (1953) 499; M. Gell-Mann and F.E. Low, Phys. Rev. 95 (1954) 1300; N.N. Bogoliubov and D.V. Shirkov, Dokl. Acad. Nauk SSSR 103 (1955) 203, 391; L.V. Ovsyannikov, Dokl. Acad. Nauk SSSR 109 (1956) 1112; K. Symanzik, Comm. Math. Phys. 18 (1970) 227; C.G. Callan Jr, Phys. Rev. D 2 (1970) 1541.

[14] G. 't Hooft, Nucl. Phys. B 61 (1973) 455. 
[15] S. Weinberg, Phys. Rev. D 8 (1973) 3497.

[16] J. Chay, H. Georgi and B. Grinstein, Phys. Lett. B 247 (1990) 399.

[17] I.I. Bigi, N.G. Uraltsev and A.I. Vainshtein, Phys. Lett. B 293 (1992) 430 [E: B 297 (1993) 477]. I.I. Bigi, M.A. Shifman, N.G. Uraltsev and A.I. Vainshtein, Phys. Rev. Lett. 71 (1993) 496; B. Blok, L. Koyrakh, M.A. Shifman and A.I. Vainshtein, Phys. Rev. D 49 (1994) 3356 [E: D 50 (1994) 3572].

[18] A.V. Manohar and M.B. Wise, Phys. Rev. D 49 (1994) 1310.

[19] Z. Ligeti and A.V. Manohar, Phys. Lett. B 433 (1998) 396; I. Bigi, M. Shifman, N. Uraltsev and A. Vainshtein, hep-ph/9805241; B.Grinstein and R.F. Lebed, hepph/9805404; N. Isgur, hep-ph/9811377.

[20] T. Inami and C.S. Lim, Progr. Theor. Phys. 65 (1981) 297.

[21] G. Buchalla, A.J. Buras and M. Lautenbacher, Rev. Mod. Phys 68 (1996) 1125.

[22] A.J. Buras and R. Fleischer, hep-ph/9704376, page 65 in [24.

[23] A.J. Buras, hep-ph/9806471, to appear in Probing the Standard Model of Particle Interactions, eds. F. David and R. Gupta (Elsevier Science B.V., Amsterdam, 1998).

[24] A.J. Buras and M. Lindner, Heavy Flavours II, World Scientific, 1998.

[25] G. Buchalla, A.J. Buras and M.K. Harlander, Nucl. Phys. B 349 (1991) 1.

[26] G. Buchalla, A.J. Buras, M.K. Harlander, M.E. Lautenbacher and C. Salazar, Nucl. Phys. B 355 (1991) 305.

[27] P. Cho, M. Misiak and D. Wyler, Phys. Rev. D 54 (1996) 3329.

[28] A. Ali, Th. Mannel and Ch. Greub, Zeit. Phys. C 67 (1995) 417.

[29] W.A. Bardeen, A.J. Buras, D.W. Duke and T. Muta, Phys. Rev. D 18 (1978) 3998.

[30] A.I. Vainshtein, V.I. Zakharov, and M.A. Shifman, JTEP 45 (1977) 670. F. Gilman and M.B. Wise, Phys. Rev. D 20 (1979) 2392; B. Guberina and R. Peccei, Nucl. Phys. B 163 (1980) 289.

[31] G. Altarelli, G. Curci, G. Martinelli and S. Petrarca, Nucl. Phys. B 187 (1981) 461.

[32] A.J. Buras and P.H. Weisz, Nucl. Phys. B 333 (1990) 66. 
[33] A.J. Buras, M. Jamin, M.E. Lautenbacher and P.H. Weisz, Nucl. Phys. B 370 (1992) 69; Nucl. Phys. B 400 (1993) 37.

[34] A.J. Buras, M. Jamin and M.E. Lautenbacher, Nucl. Phys. B 400 (1993) 75.

[35] A.J. Buras, M. Jamin and M.E. Lautenbacher, Nucl. Phys. B 408 (1993) 209.

[36] M. Ciuchini, E. Franco, G. Martinelli and L. Reina, Phys. Lett. B 301 (1993) 263.

[37] M. Ciuchini, E. Franco, G. Martinelli and L. Reina, Nucl. Phys. B 415 (1994) 403.

[38] K. Chetyrkin, M. Misiak and Münz, Nucl. Phys. B520 (1998) 279.

[39] M.Misiak and M. Münz, Phys. Lett. B344 (1995) 308.

[40] K.G. Chetyrkin, M. Misiak and M. Münz, Phys. Lett. B400 (1997) 206; Erratum ibid. B425 (1998) 414.

[41] G. Buchalla, Nucl. Phys. B 391 (1993) 501.

[42] E. Bagan, P.Ball, V.M. Braun and P.Gosdzinsky, Nucl. Phys. B 432 (1994) 3; E. Bagan et al., Phys. Lett. B 342 (1995) 362; B 351 (1995) 546.

[43] A. Lenz, U. Nierste and G. Ostermaier, Phys. Rev. D 56 (1997) 7228.

[44] M. Jamin and A. Pich, Nucl. Phys. B425 (1994) 15.

[45] S. Herrlich and U. Nierste, Nucl. Phys. B419 (1994) 292.

[46] A.J. Buras, M. Jamin, and P.H. Weisz, Nucl. Phys. B347 (1990) 491.

[47] J. Urban, F. Krauss, U.Jentschura and G. Soff, Nucl. Phys. B523 (1998) 40.

[48] S. Herrlich and U. Nierste, Phys. Rev. D52 (1995) 6505; Nucl. Phys. B476 (1996) 27.

[49] G. Buchalla and A.J. Buras, Nucl. Phys. B 398 (1993) 285.

[50] G. Buchalla and A.J. Buras, Nucl. Phys. B 400 (1993) 225.

[51] M. Misiak and J. Urban, hep-ph/9901278.

[52] G. Buchalla and A.J. Buras, hep-ph/9901288.

[53] G. Buchalla and A.J. Buras, Nucl. Phys. B 412 (1994) 106.

[54] G. Buchalla and A.J. Buras, Phys. Lett. B 336 (1994) 263. 
[55] A. J. Buras, M. E. Lautenbacher, M. Misiak and M. Münz, Nucl. Phys. B423 (1994) 349.

[56] M. Misiak, Nucl. Phys. B393 (1993) 23; Erratum, Nucl. Phys. B439 (1995) 461.

[57] A.J. Buras and M. Münz, Phys. Rev. D 52 (1995) 186.

[58] A. Ali, and C. Greub, Z.Phys. C49 (1991) 431; Phys. Lett. B259 (1991) 182; Phys. Lett. B361 (1995) 146.

[59] K. Adel and Y.P. Yao, Modern Physics Letters A8 (1993) 1679; Phys. Rev. D 49 (1994) 4945.

[60] N. Pott, Phys. Rev. D 54 (1996) 938.

[61] C. Greub, T. Hurth and D. Wyler, Phys. Lett. B380 (1996) 385; Phys. Rev. D 54 (1996) 3350;

[62] C. Greub and T. Hurth, Phys. Rev. D 56 (1997) 2934; hep-ph/9809468.

[63] A.J. Buras, A. Kwiatkowski and N. Pott, Phys. Lett. B 414 (1997) 157, Nucl. Phys. B 517 (1998) 353.

[64] M. Ciuchini, G. Degrassi, P. Gambino and G.F. Giudice, Nucl. Phys. B 527 (1998) 21.

[65] F.M. Borzumati and Ch. Greub, Phys. Rev. D 58 (1998) 07004; hep-ph/9809438.

[66] P. Ciafaloni, A. Romanino, and A. Strumia, Nucl. Phys. B 524 (1998) 361.

[67] M. Beneke, G. Buchalla, C. Greub, A. Lenz and U. Nierste, hep-ph/9808385.

[68] M. Beneke, F. Maltoni and I.Z. Rothstein, hep-ph/9808360.

[69] M. Ciuchini, E. Franco, V. Lubicz, G. Martineli, I. Scimeni and L. Silvestrini, Nucl. Phys. B 523 (1998) 501.

[70] M. Ciuchini, et al. JHEP 9810 (1998) 008.

[71] M. Ciuchini, G. Degrassi, P. Gambino and G.F. Giudice, Nucl. Phys. B 534 (1998) 3.

[72] G. Buchalla and A.J. Buras, Phys. Rev. D57 (1998) 216.

[73] A. Czarnecki and W.J. Marciano, Phys. Rev. Lett. 81 (1998) 277.

[74] A. Strumia, Nucl. Phys. B532 (1998) 28. 
[75] A.L. Kagan and M. Neubert, hep-ph/9805303.

[76] P. Gambino, A. Kwiatkowski and N. Pott, hep-ph/9810400.

[77] K. Chetyrkin, M. Misiak and Münz, Nucl. Phys. B518 (1998) 473.

[78] S. Herrlich and U. Nierste, Nucl. Phys. B 445 (1995) 39.

[79] J. Alexander; D. Karlen; P. Rosnet; talks given at the 29th International Conference on High Energy Physics (ICHEP 98), Vancouver, Canada, 23-29 July 1998, to appear in the proceedings.

[80] Particle Data Group, Phys. Rev. D 54 (1996) 1.

[81] A.J. Buras, M.E. Lautenbacher and G. Ostermaier, Phys. Rev. D 50 (1994) 3433.

[82] L. Wolfenstein, Phys. Rev. Lett. 51 (1983) 1945.

[83] L. Wolfenstein, Phys. Rev. Lett. 13 (1964) 562.

[84] J. M. Flynn and L. Randall, Phys. Lett. B224 (1989) 221; erratum ibid. Phys. Lett. B235 (1990) 412.

[85] G. Buchalla, A. J. Buras, and M. K. Harlander, Nucl. Phys. B337 (1990) 313.

[86] R. Gupta, hep-ph/9801412; R.S. Sharpe, hep-lat/9811006.

[87] G. D. Barr et al., Phys. Lett. B317 (1993) 233.

[88] L. K. Gibbons et al., Phys. Rev. Lett. 70 (1993) 1203.

[89] A. Ali, and C. Greub, Z.Phys. C60 (1993) 433.

[90] A. J. Buras, M. Misiak, M. Münz and S. Pokorski, Nucl. Phys. B424 (1994) 374.

[91] L. Littenberg, Phys. Rev. D39 (1989) 3322.

[92] G. Buchalla and A.J. Buras, Phys. Lett. B333 (1994) 221; Phys. Rev. D54 (1996) 6782 .

[93] W. Marciano and Z. Parsa, Phys. Rev. D53, R1 (1996).

[94] D. Rein and L.M. Sehgal, Phys. Rev. D39 (1989) 3325; J.S. Hagelin and L.S. Littenberg, Prog. Part. Nucl. Phys. 23 (1989) 1; M. Lu and M.B. Wise, Phys. Lett. B324 (1994) 461; S. Fajfer, Nuovo Cim. 110A (1997) 397; C.Q. Geng, I.J. Hsu and Y.C. Lin, Phys. Rev. D54 (1996) 877. 
[95] G. Buchalla and G. Isidori, hep-ph/9806501.

[96] S. Adler et al., Phys. Rev. Lett. 79, (1997) 2204.

[97] J. Adams et al., hep-ex/9806007.

[98] Y. Grossman, Y. Nir and R. Rattazzi, hep-ph/9701231] in [24]; Y. Grossman and Y. Nir, Phys. Lett. B398 (1997) 163;

[99] L. Littenberg and J. Sandweiss, eds., AGS2000, Experiments for the 21st Century, BNL 52512 .

[100] P. Cooper, M. Crisler, B. Tschirhart and J. Ritchie (CKM collaboration), EOI for measuring $\operatorname{Br}\left(K^{+} \rightarrow \pi^{+} \nu \bar{\nu}\right)$ at the Main Injector, Fermilab EOI 14, 1996.

[101] K. Arisaka et al., KAMI conceptual design report, FNAL, June 1991.

[102] T. Inagaki, T. Sato and T. Shinkawa, Experiment to search for the decay $K_{L} \rightarrow \pi^{0} \nu \bar{\nu}$ at KEK $12 \mathrm{GeV}$ proton synchrotron, 30 Nov. 1991.

[103] R. Fleischer, Int. J. of Mod. Phys. A12 (1997) 2459.

[104] W. A. Bardeen, A. J. Buras and J.-M. Gérard, Phys. Lett. B192 (1987) 138; A. Pich and E. de Rafael, Nucl. Phys. B358 (1991) 311; M. Neubert and B. Stech, Phys. Rev. D 44 (1991) 775; M. Jamin and A. Pich, Nucl. Phys. B425 (1994) 15; J. Kambor, J. Missimer and D. Wyler, Nucl. Phys. B346 (1990) 17; Phys. Lett. B261 (1991) 496; V. Antonelli, S. Bertolini, M. Fabrichesi, and E.I. Lashin, Nucl. Phys. B469 (1996) 181; J. Bijnens and J. Prades, hep-ph/9811472.

[105] M. Neubert and B. Stech, hep-ph/9705292, page 294 in 24]; B. Stech hepph/9706384; M.Neubert, Nucl. Phys. B (Proc. Suppl.) 64 (1998) 474, hep$\mathrm{ph} / 9801269]$.

[106] A.J. Buras and L. Silvestrini, hep-ph/9812392.

[107] A. Ali, hep-ph/9812434. 
Table 1: References to NLO Calculations

\begin{tabular}{|c|c|}
\hline Decay & Reference \\
\hline \multicolumn{2}{|l|}{$\Delta F=1$ Decays } \\
\hline current-current operators & 31,32 \\
\hline QCD penguin operators & $33,35,36,37,38$ \\
\hline electroweak penguin operators & {$[34, \overline{35}, \overline{36}, \overline{37}$} \\
\hline magnetic penguin operators & 39,40 \\
\hline $\operatorname{Br}(B)_{S L}$ & {$[31, \overline{41}, 42,43$} \\
\hline inclusive $\Delta S=1$ decays & 44] \\
\hline \multicolumn{2}{|c|}{ Particle-Antiparticle Mixing } \\
\hline$\eta_{1}$ & 45 \\
\hline$\eta_{2}, \eta_{B}$ & 46,47 \\
\hline$\eta_{3}$ & 48 \\
\hline \multicolumn{2}{|c|}{ Rare $K$ - and $B$-Meson Decays } \\
\hline$K_{L}^{0} \rightarrow \pi^{0} \nu \bar{\nu}, B \rightarrow l^{+} l^{-}, B \rightarrow X_{\mathrm{s}} \nu \bar{\nu}$ & $49,50,51,52$ \\
\hline$K^{+} \rightarrow \pi^{+} \nu \bar{\nu}, K_{\mathrm{L}} \rightarrow \mu^{+} \mu^{-}$ & 53,52 \\
\hline$K^{+} \rightarrow \pi^{+} \mu \bar{\mu}$ & [54] \\
\hline$K_{\mathrm{L}} \rightarrow \pi^{0} e^{+} e^{-}$ & [55] \\
\hline$B \rightarrow X_{s} \mu^{+} \mu^{-}$ & {$[56,57$} \\
\hline$B \rightarrow X_{s} \gamma$ & [58]-65] \\
\hline$\Delta \Gamma_{B_{s}}$ & 67 \\
\hline inclusive $\mathrm{B} \rightarrow$ Charmonium & 68 \\
\hline \multicolumn{2}{|c|}{ Two-Higgs Doublet Models } \\
\hline$B \rightarrow X_{s} \gamma$ & $64,66,65$ \\
\hline \multicolumn{2}{|l|}{ Supersymmetry } \\
\hline$\Delta M_{K}$ and $\varepsilon_{K}$ & 69,70 \\
\hline$B \rightarrow X_{s} \gamma$ & [71] \\
\hline
\end{tabular}

Table 2: Electroweak Two-Loop Calculations

\begin{tabular}{|l|l|}
\hline \multicolumn{1}{|c|}{ Decay } & Reference \\
\hline$K_{L}^{0} \rightarrow \pi^{0} \nu \bar{\nu}, B \rightarrow l^{+} l^{-}, B \rightarrow X_{\mathrm{s}} \nu \bar{\nu}$ & 72 \\
$B \rightarrow X_{s} \gamma$ & 73, 74, 75 \\
$B^{0}-\bar{B}^{0}$ mixing & 76 \\
\hline
\end{tabular}

